\title{
Hypoplasia of the Parafacial/Facial Complex: A Very Frequent Finding in Sudden Unexplained Fetal Death
}

\author{
Anna Maria Lavezzi* and Luigi Matturri
}

\author{
Institute of Pathology, “Lino Rossi” Research Center, University of Milan, Italy
}

\begin{abstract}
AIMS - To define firstly in man the localization and the anatomical boundaries of the parafacial respiratory group in the brainstem. Thereafter, to determine whether this center, given its essential role in the respiratory rhythmgenerating circuit, showed abnormalities in sudden unexplained perinatal and infant deaths, like other nuclei and/or structures of the brainstem and cerebellum checking vital functions, that we have previously reported.
\end{abstract}

METHODS - In 67 brains collected from 29 stillbirths, 9 newborns and 29 infants, died of both known and unknown cause, an in-depth histological examination of the autonomic nervous system was made, according to the protocol routinely followed by the Institute of Pathology, University of Milan. In particular we analyzed the parafacial and facial nuclei in serial sections of caudal pons.

RESULTS - We firstly identified and defined the normal structure of the parafacial/facial complex in control cases. Besides we diagnosed the hypoplasia of these nuclei in $75 \%$ of sudden unexplained fetal deaths and never after birth.

CONCLUSIONS - We formulate the hypothesis that the hypoplasia of the parafacial/facial complex is a specific marker of unexplained stillbirth, and that the normal development of this complex is essential for extra-uterine life.

Keywords: Neuropathology, stillbirth, facial nucleus, parafacial nucleus, hypoplasia.

\section{INTRODUCTION}

In the course of our neuropathological studies, an impressive body of evidence has accumulated stressing the relevance of a neuronal network extending from the hindbrain (that includes medulla oblongata, pons and cerebellum) to the midbrain. This network acts in unison to support the vital processes, and even its minute alterations can lead to sudden unexplained perinatal death and/or sudden infant death [1-8].

Hypoplasia of different brainstem nuclei, particularly of the arcuate nucleus, is frequently observed in these pathologies. Recently we have also identified hypoplasia of the preBötzinger complex [9], a structure of the ventrolateral medulla that has been demonstrated in experimental studies performed on rats to be essential for the generation of the respiratory rhythm [10-13].

A consensus is now emerging in the literature that respiratory rhythm does not derive from this single medullary site, but also from a second, more rostral neuronal center. This is referred to as the "parafacial respiratory group", due to its position related to the facial nucleus [14-17]. The related neurons, defined as "pre-inspiratory neurons", trigger the inspiratory function in the pre-Bötzinger complex. So, the latest view is that the parafacial respiratory group is the master generator of the respiratory rhythm in mammals. Nevertheless, this neuronal center has never previously been investigated in human neuropathologic research.

In the present study, we firstly sought to define the localization and trace the anatomical boundaries of the parafacial respiratory group in the human brainstem. Thereafter, we

\footnotetext{
*Address correspondence to this author at the Institute of Pathology, University of Milan, Via della Commenda, 19, 20122 Milan, Italy; Tel:+39-0250320821; Fax: +39-02-50320823; E-mail: anna.lavezzi@unimi.it
}

aimed to determine whether this center, given its essential role in the respiratory rhythm-generating circuit, showed abnormalities in sudden unexplained perinatal and infant deaths, like other nuclei and/or structures of the brainstem and cerebellum checking vital functions, that we have reported in previous works [1-8].

\section{MATERIAL AND METHODS}

A total of 67 brains were collected from 29 fresh stillbirths (22-40 gestational weeks, with a peak from 36 to 40 weeks), 9 newborns who died within the first week of life and 29 infants aged 1-10 months (mean age: 3 months).

The victims were subjected to a complete autopsy, including examination of the placental disk, umbilical cord and membranes in fetuses. In all cases an in-depth histological examination of the autonomic nervous system was made, according to the protocol routinely followed by the Institute of Pathology, University of Milan [18,19].

In particular, after fixation in $10 \%$ phosphate-buffered formalin, the brainstem, main objective of this study, was processed and embedded in paraffin.

Precisely, the examination of the brainstem includes the sampling of three specimens. The first specimen, pontomesencephalic, includes the upper third of the pons and the adjacent portion of midbrain. The second extends from the upper third of the medulla oblongata to the portion adjacent to the pons. The third specimen takes as reference point the obex and extends 2-3 $\mathrm{mm}$ above it and below it.

Transverse serial sections of the midbrain, pons and medulla oblongata were made in each of these three samples at intervals of $30 \mu \mathrm{m}$. For each level, twelve $5 \mu \mathrm{m}$ sections were obtained, two of which were routinely stained for histological examination using hematoxylin-eosin and Klüver- 
Table 1. Case Profiles in the Study

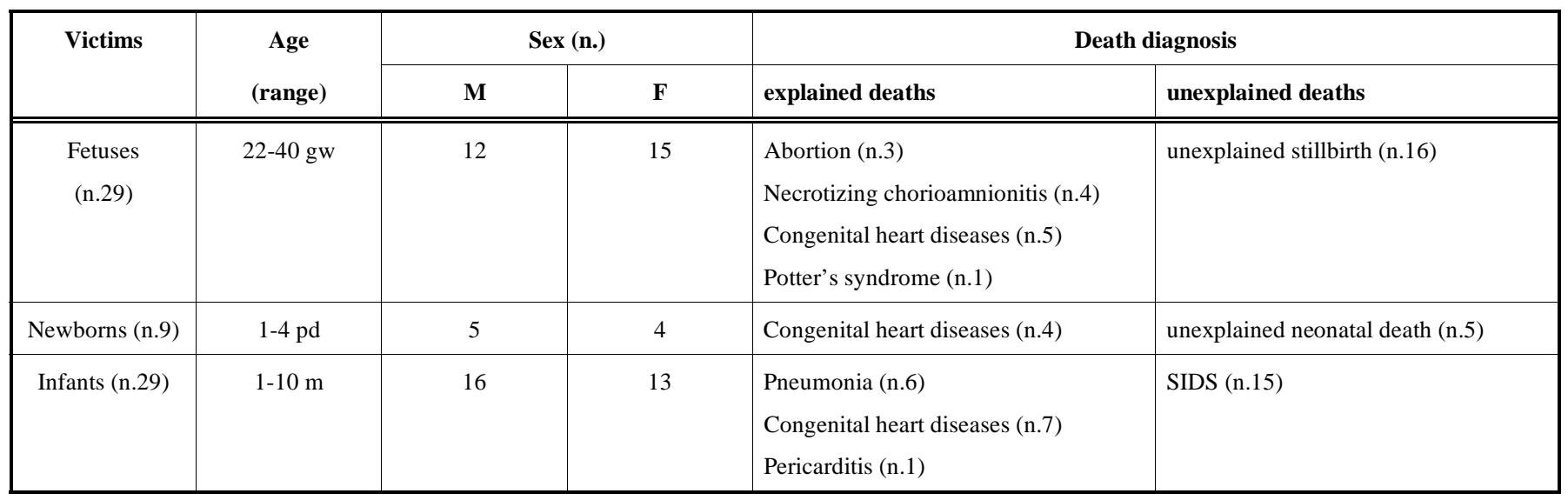

$\mathrm{gw}=$ gestational week; $\mathrm{pd}=$ postnatal day $; \mathrm{m}=$ month .

SIDS $=$ Sudden Infant Death Syndrome.

Barrera. The remaining sections were saved and stained as deemed necessary for further investigations.

The routine histological evaluation of the brainstem was focused on the locus coeruleus and on the parabrachial/Kölliker-Fuse complex in the pons/mesencephalon, and on the hypoglossus, the dorsal motor vagal, the tractus solitarius, the ambiguus, the pre-Bötzinger, the inferior olivary and the arcuate nuclei in the medulla oblongata. In addition, for the first time we also defined and analyzed the parafacial and facial nuclei in serial sections of caudal pons.

In 36 cases, after the in-depth autoptic examination, the death remained totally unexplained. A diagnosis of "unexplained stillbirth" was established for 16 fetuses, who died suddenly after the $22^{\text {nd }}$ gestational week before complete expulsion or retraction from the mother; a diagnosis of "unexplained neonatal death" for 5 newborns who died between birth and the end of the $1^{\text {st }}$ postnatal week, and of SIDS for 15 infants died within the first year of life. In the remaining 31 cases, 13 stillbirths, 4 neonatal deaths and 14 infant deaths, a precise cause of death was formulated at autopsy. Therefore, these cases were regarded as "controls".

Table 1 summarizes the case profiles in this study, indicating the sex distribution, range of ages and death diagnoses.

\section{Statistical Analysis}

The statistical significance of direct comparison between the groups of victims was determined using analysis of variance (ANOVA). Statistical calculations were carried out on a personal computer with SPSS statistical software. The selected threshold level for statistical significance was $\mathrm{P}<0.05$.

\section{RESULTS}

Firstly, we proceeded to identify the localization and the morphology of the parafacial group, the target of this study, in the brainstem of the 31 control cases, following the indications provided by experimental studies in this field. All these reports showed the parafacial nucleus to lie ventral and lateral to the facial nucleus.

The facial nucleus is easily identifiable in humans, and is seen in transversal sections of caudal pons at the source of the homonymous nerve (Fig. 1). It appears as a roundish cluster of large polygonal neurons with evident axons and dendrites, a light central nucleus with a marked nucleolus, an abundant cytoplasm rich in Nissl substance, adjacent to the trapezoid nucleus and, in the more caudal sections at the boundaries of the brainstem, adjacent to the upper olivary nucleus.
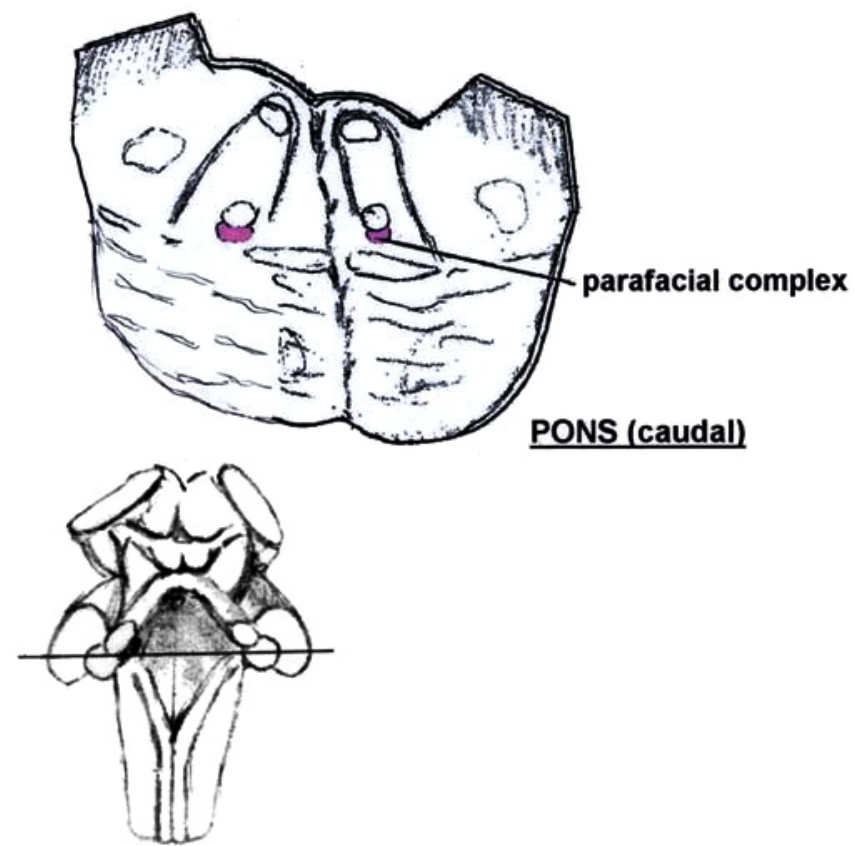

Fig. (1). Schematic representation of the histological sections obtained from the caudal pons with the localization of the parafacial complex.

The human parafacial group, that we defined in a simple way as "parafacial nucleus", ventro-laterally surrounds the facial nucleus. Compared with the facial nucleus neurons, the parafacial neurons are similar in shape but more intensely stained in both hematoxylin-eosin and Klüver-Barrera preparations, due to an increased number of pigment granules in the cytoplasm. Frequently, the neurons of the two nuclei are inter-mixed. Thus, we defined these two nuclei taken together as the "parafacial/facial complex" (pF/Fc) (Figs. 2 and 3). 
After defining the anatomical localization of the parafacial and facial nuclei in the control cases, we examined this neuronal complex in the 36 victims of the study who died without an explained cause (16 unexplained stillbirths, 5 unexplained neonatal deaths and 15 SIDS).

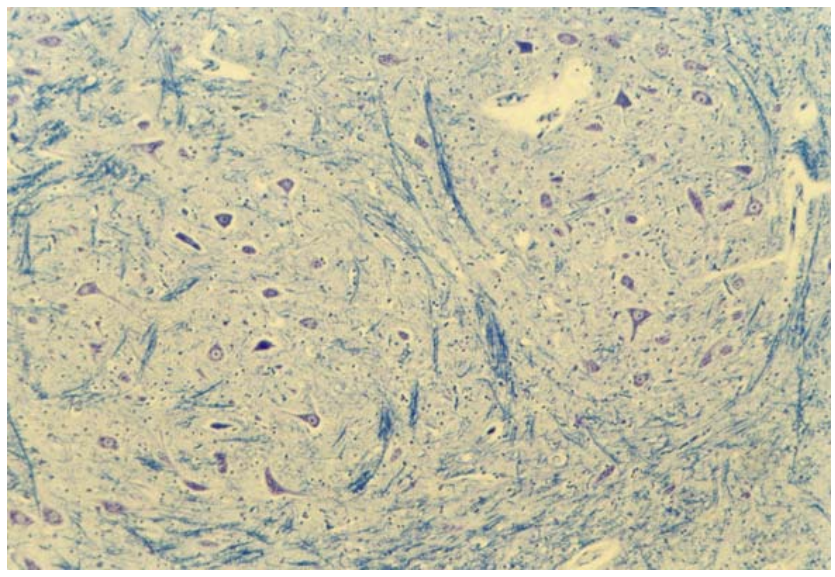

Fig. (2). Facial/Parafacial Complex.

Fig. (2) shows the facial and parafacial nuclei in a case of sudden intrauterine unexplained death of 36 gestational weeks (histological section of caudal pons - Klüver-Barrera stain - Magnification 20x). The neurons of the parafacial nucleus are more intensely stained. This aspect is better evident at increased magnification (40x) in Fig. (3).

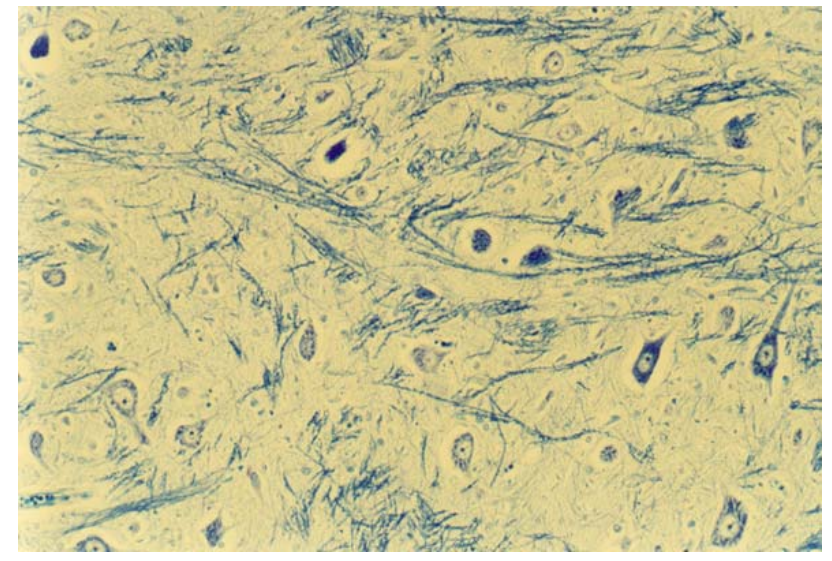

Fig. (3). Facial/Parafacial Complex.

Hypoplasia of both the facial and parafacial nuclei, with decreased neuronal density and transversal area, was diagnosed in 12 cases of unexplained fetal death $(75 \%$ of the unexplained stillbirths). The neuronal cell bodies of the hypoplasic nuclei were sometimes morphologically similar to the neurons of control cases but more frequently, they were smaller and lengthened with a flattened nucleus, compact chromatin and a less evident nucleolus (Fig. 4).

Histological examination of the other brainstem nuclei throughout their extension showed different developmental abnormalities: hypoplasia of the arcuate nucleus in 14 of the 34 sudden death victims (6 fetal, 1 neonatal death and 7 SIDS victims) and in 4 of the 31 control subjects who died of other causes; hypoplasia of the pre-Bötzinger nucleus was observed in 5 unexplained stillbirths, 2 unexplained neonatal deaths and 3 SIDS and in 2 control cases; hypoplasia of the
parabrachial/Kölliker-Fuse complex was present in 4 unexplained fetal death victims and 2 unexplained neonatal deaths; hypoplasia of the hypoglossus nucleus, in two SIDS cases.

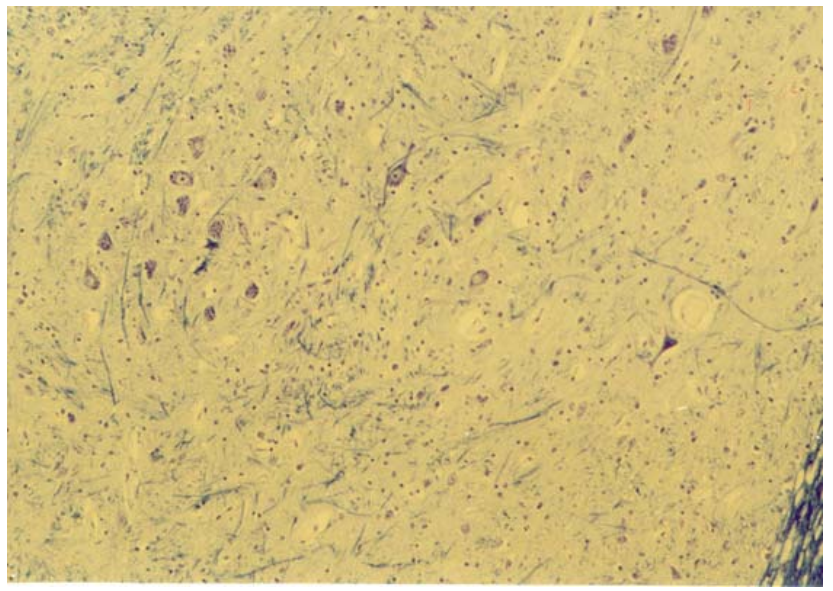

Fig. (4). Hypoplasia of the Facial/Parafacial Complex in a case of sudden intrauterine unexplained death of 38 gestational weeks (histological section of caudal pons - Klüver-Barrera stain - Magnification 20x).

Table 2 summarizes the distribution of the hypoplasic nuclei observed in this study in both unexplained and explained deaths.

Besides, in three cases of unexplained fetal death, hypoplasia of the $\mathrm{pF} / \mathrm{Fc}$ was associated with pre-Bötzinger and arcuate nuclei hypoplasia.

\section{DISCUSSION}

For many years the prevailing theory has been that the mammalian respiratory network has a single rhythmogenic kernel that controls the timing of inspiration, expiration and airway muscle activity in the ventrolateral medulla named the "pre-Bötzinger complex" [9-13].

However, the autonomy of this complex in respiratory rhythm generation has recently been questioned by a series of experiments that identified a second, more important "neuronal respiratory rhythm generator" located more rostrally, adjacent to the facial nucleus [14-17].

In particular, supporting the theory of two distinct respiratory centers in the brainstem, Onimaru et al. [17] provided the basis for the hypothesis that the pre-Bötzinger complex is the rhythm generator for inspiratory activity, whereas the generator of rhythmic expiratory activity is located in the parafacial nucleus. Moreover, their findings allowed them to define the neurons of the parafacial respiratory group as "pre-inspiratory" neurons, because these neurons periodically trigger the inspiratory neurons of the pre-Bötzinger complex. Thus, the pre-Bötzinger complex appears to be activated by the parafacial respiratory group, in other words, inspiratory activity is always preceded by firing of the preinspiratory neurons. Therefore, the authors ascribed the function of hierarchical modulation of the medullary breathing circuitry in mammals to the parafacial respiratory group.

The present study is the first to identify the anatomic localization and the cytoarchitecture of the parafacial respiratory group in humans. In fact, in a wide sample of fetal and 
Table 2. Distribution of the Neuropathologic Findings in Victims of Perinatal and Infant Death

\begin{tabular}{|c|c|c|c|c|c|c|}
\hline $\begin{array}{c}\text { morphologic alterations } \\
\text { in the brainstem }\end{array}$ & $\begin{array}{c}\text { unexplained } \\
\text { stillbirth }\end{array}$ & control stillbirth & $\begin{array}{c}\text { unexplained neo- } \\
\text { natal death }\end{array}$ & $\begin{array}{c}\text { control neonatal } \\
\text { death }\end{array}$ & $\begin{array}{c}\text { SIDS } \\
\text { control infant } \\
\text { death }\end{array}$ \\
\hline \hline pF/Fc hypoplasia & $12^{* *}$ & - & - & - & - \\
\hline ArcN hypoplasia & $6^{*}$ & 1 & 1 & - & $7^{*}$ & 3 \\
\hline pBN hypoplasia & $5^{*}$ & 1 & 2 & - & - \\
\hline PB/KFc hypoplasia & $4^{*}$ & - & - & - & - \\
\hline hypoglN hypoplasia & - & - & - & - & -2 \\
\hline
\end{tabular}

ArcN = arcuate nucleus; HypoglN = hypoglossus nucleus; $\mathrm{pBN}=$ pre-Bötzinger nucleus; $\mathrm{PB} / \mathrm{KFc}=$ parabrachial/ Kölliker-Fuse complex; $\mathrm{pF} / \mathrm{Fc}=$ parafacial/facial complex . Significance as compared to controls (explained deaths) is expressed for $* \mathrm{P}<0.05$, $* * \mathrm{P}<0.01$.

infant victims who died of known causes, we defined a cluster of neurons in the caudal pons surrounding the facial nucleus, that we regarded simply as the "parafacial nucleus", rather than the parafacial group. Since the neurons of the parafacial nucleus are frequently inter-mixed with the neurons of the facial nucleus, we called the whole neuronal group the "parafacial/facial complex" $(\mathrm{pF} / \mathrm{Fc})$.

The association of these two nuclei in the same neuronal complex may seem incoherent. In fact, while the involvement of the parafacial nucleus in the primary generation of the respiratory rhythm has been demonstrated [14-17], the facial nucleus is traditionally considered as a motor nucleus that controls the activity of the facial muscles through its peripheral efferents [20-22].

Some reports have shown, however, that there are some non-motor neurons or interneurons in the facial nucleus that have axonal projections to different brainstem respiratory nuclei, namely to the tractus solitarius nucleus, the ambiguus nucleus in the medulla oblongata and to the parabrachial and Kolliker-Fuse nuclei in the pons [23,24]. Besides, Zhang et al. [25] found in experimental studies in rats that some neurons in the facial nucleus were retrogradely labeled by biocytin injected into the pre-Bötzinger complex region, confirming that the facial nucleus takes part in the regulation of the respiratory activity.

Therefore, damage to neurons of the parafacial-facial complex could contribute to respiratory rhythm perturbation.

In this study we diagnosed a very high frequency of hypoplasia of the $\mathrm{pF} / \mathrm{Fc}$, in terms of a decreased neuronal body number and area of both facial and parafacial nuclei, exclusively in unexplained fetal deaths $(75 \%$ of all unexplained stillbirths) but in no case that died after birth.

It is reasonable to speculate whether breathing alterations can lead to death during intrauterine life. It is known that the respiratory-related neuronal network is active before birth and controls respiratory-like rhythmic movements that are among the earliest detectable behavior of the mammalian fetus. Therefore, it becomes vital only after birth.

Thus, an immature respiratory activity is demonstrable in prenatal stages, shortly after the onset of fetal movements. Consequently, the observed morpho-functional alterations of the central autonomic nervous system, and particularly the great frequency of hypoplasia of the $\mathrm{pF} / \mathrm{Fc}$ in sudden unexplained stillbirth, could determine defects of this occasional respiratory activity in prenatal life. This is supported by the frequent finding of lung hypoplasia in fetuses [26].

Nevertheless, these breathing alterations would not be sufficient to justify fetal death. One possibility is that the neuronal structures involved participate not only in respiratory modulation but, more extensively, are essential to the control of other vital functions.

Another hypothesis could be, however, that these nuclei really are appointed only to respiratory control, but that in the last weeks of pregnancy, advancing towards the time of birth, a general check of all the essential functions for extrauterine life occurs, particularly of the respiratory activity. Sudden unexpected fetal death could therefore be ascribed to a selective process of natural suppression in presence of developmental alterations of the respiratory network, and particularly of hypoplasia of the $\mathrm{pF} / \mathrm{Fc}$, that is present in $75 \%$ of cases.

In conclusion, in view of the exclusive presence of hypoplasia of the $\mathrm{pF} / \mathrm{Fc}$ in the prenatal stages, we formulate the hypothesis that this developmental alteration is a specific marker of unexplained stillbirth, or at least of a major subset of cases of unexplained stillbirth. Failure to observe this alteration after birth is indicative of the essential importance of normal development of the $\mathrm{pF} / \mathrm{Fc}$ for extra-uterine life.

\section{ACKNOWLEDGMENTS}

This study was supported by the Italian Lombardy Region target project n. 49210/2000 (Program of research and intervention for the reduction of the risk of SIDS and unexpected fetal death), by the agreement Lombardy RegionPfizer Italia S.r.1. n. 814/2006, and by Ministry of Foreign Affairs (joined projects of particular relevance "Anatomopathologic and genetic study of the unexplained perinatal death and SIDS") n. 269/P/0085087/2004 and n. 0083227/20006. The authors thank Mary Victoria Candace Pragnell, B.A. for English language assistance.

\section{ABBREVIATIONS}

$\begin{array}{lll}\mathrm{pF} / \mathrm{Fc} & = & \text { Parafacial/facial complex } \\ \text { ArcN } & = & \text { Arcuate nucleus } \\ \text { HypoglN } & =\text { Hypoglossus nucleus } \\ \mathrm{pBN} & =\text { Pre-Bötzinger nucleus } \\ \mathrm{PB} / \mathrm{KFc} & =\text { Parabrachial/ Kölliker-Fuse complex }\end{array}$




\section{REFERENCES}

[1] Lavezzi AM, Ottaviani G, Mauri M, Matturri L. Hypoplasia of the arcuate nucleus and maternal smoking during pregnancy, in perinatal and infant sudden unexpected death. Neuropathology 2003; 24: 284-289.

[2] Lavezzi AM, Ottaviani G, Matturri L. Role of somatostatin and apoptosis in breathing control in sudden perinatal and infant unexplained death. Clin Neuropathol 2004; 23: 304-310.

[3] Lavezzi AM, Ottaviani G, Ballabio GM, Rossi L, Matturri L. Preliminary study on the cytoarchitecture of the human parabrachial/Kölliker-Fuse complex with reference to sudden infant death syndrome and sudden intrauterine unexplained death. Pediatr Dev Pathol 2004; 7: 171-179.

[4] Lavezzi AM, Ottaviani G, Mingrone R, Matturri L. Analysis of the human locus coeruleus in perinatal and infant sudden unexplained deaths. Possible role of cigarette smoking in the development of this nucleus. Brain Res Dev Brain Res 2005; 154: 71-80.

[5] Lavezzi AM, Ottaviani G, Matturri L. Adverse effects of prenatal tabacco smoke exposure on biological parameters of the developing brainstem. Neurobiol Dis 2005; 20: 601-607.

[6] Lavezzi AM, Ottaviani G, Mauri M, Matturri L. Alterations of biological features of the cerebellum in sudden perinatal and infant death. Curr Mol Med 2006; 6: 429-435.

[7] Matturri L, Minoli I, Lavezzi AM, Cappellini A, Ramos S, Rossi L. Hypoplasia of medullary arcuate nucleus in unexpected late fetal death (stillborn infants): a pathologic study. Pediatrics 2002; 109: $1-5$.

[8] Lavezzi AM, Ottaviani, Matturri L. Involvement of somatostatin in breathing control before and after birth, and in perinatal and infant sudden unexplained death. Folia Neuropathol 2004; 42: 59-65

[9] Lavezzi AM, Matturri L. Functional neuroanatomy of the human pre-Botzinger complex with particular reference to sudden unexplained perinatal and infant death. Neuropathology 2007 (in press).

[10] Duffin J, Ezure K, Lipski J. Breathing rhythm generation: focus on the rostral ventrolateral medulla. News Physiol Sci 1995; 10: 133140.

[11] McKay LC, Janczewski WA, Feldman JL. Sleep-disordered breathing after targeted ablation of preBötzinger complex neurons. Nat Neurosci 2005; doi 10.1038/nn1517.

[12] Rekling JC, Feldman JL. PreBötzinger complex and pacemaker neurons: hypothesized site and kernel for respiratory rhythm generation. Annu Rev Physiol 1998; 60: 385-405.

[13] Smith JC, Ellenberger HH, Ballanyi K, Richter DW, Feldman JL. PreBötzinger complex: a brainstem region that may generate respiratory rhythm in mammals: Science 1991; 254: 726-729.
[14] Janczewski WA, Feldman JL. Distinct rhythm generators for inspiration and expiration in the juvenile rat. J Physiol 2006; 570: 407420.

[15] Chatonnet F, Borday C, Wrobel L, Thoby-Brissoon M, Fortin G, McLean H, Champagnat J. Ontogeny of central rhythm generation in chicks and rodents. Respir Physiol 2006; 154: 37-56.

[16] Guyenet PG. Novel two-rhythm generator theory of breathing in mammals. J Physiol 2006; 570: 407-420.

[17] Onimaru H, Kumagawa, Homma I. Respiration-related rhythmic activity in the rostral medulla of newborn rats. J Neurophysiol 2006; 96: 55-61.

[18] Matturri L, Ottaviani G, Alfonsi G, Crippa M, Rossi L, Lavezzi AM. Study of the brainstem, particularly the arcuate nucleus, in sudden infant death syndrome (SIDS) and sudden intrauterine unexplained death (SIUD). Am J Forensic Med Pathol 2004; 25: 4448.

[19] Matturri L, Ottaviani G, Lavezzi AM. Techniques and criteria in pathologic and forensic-medical diagnostics of sudden unexpected infant and perinatal death. Am J Clin Pathol 2005; 124: 259-268.

[20] Sherwood CC. Comparative anatomy of the facial motor nucleus in mammals, with ana analysis of neuron numbers in primates. Anat Rec A Discov Mol Cell Evol Biol 2005; 287: 1067-1079.

[21] Horta JA, Tamega OJ, Cruz-Rizzolo RJ. Cytoarchitecture and musculotopic organization of the facial motor nucleus in Cebus apella monkey. J Anat 2004; 204: 175-190.

[22] Furutani R, Sugita S. Projection from the facial nucleus to the auricular muscles of rabbits. Okajimas Folia Anat Jpn 2004; 81: $109-118$

[23] Li C, Guan ZL, Chan YS, Zheng Y. Projections from facial nucleus interneurons to the respiratory groups of brainstem in the rat. Neurosci Lett 2004; 4: doi 10.1016/j.neulet.2004.06.080.

[24] Bendet E, Vajtai I, Maranta C, Fish U. Rate and extent of early axonal degeneration of the human facial nerve. Prog Biophys Mol Biol 2004; 84: 89-94.

[25] Zhang C, Yan H, Li C, Zheng Y. Possible involvement of the facial nucleus in regulation of respiration in rats. Neurosci Lett 2004; 367: 283-288

[26] Matturri L., Lavezzi AM, Cappellini A, Ottaviani G., Minoli I, Rubino B, Rossi L. Association between pulmonary hypoplasia and hypoplasia of arcuate nucleus in stillbirth. J Perinatol 2003; 23: 328-332. 